\title{
¿Revistas 2.0? Revistas científicas españolas del área de Comunicación en las redes sociales ${ }^{1}$
}

\author{
Francisco SEgADO-BoJ \\ Universidad Internacional de la Rioja \\ francisco.segado@unir.net
}

Recibido: $05 / 10 / 2012$

Aceptado: 23/01/2013

\begin{abstract}
Resumen
Este trabajo analiza los perfiles de las revistas académicas indexadas en el In-Recs de 2010 en las redes sociales más usadas en España (Twitter y Facebook). En concreto pretende comprobar la presencia de estas publicaciones estas redes. Otro objetivo es medir el grado de popularidad de estas revistas, estimado en número de seguidores o de fans. Se comprueba de este modo que, salvo excepciones, las principales revistas científicas del campo muestran un desinterés por estas herramientas de divulgación del conocimiento.

Palabras clave: Comunicación, Facebook, Revistas académicas, redes sociales, Twitter

\section{Journals 2.0? Communication Area Spanish Academic Journals in Social Networking Sites}

\begin{abstract}
This paper analyses the profiles of the academic journals indexed at the 2010 edition of In-recs in the most popular social networking sites in Spain (Twitter and Facebook). Thus the presence of these publications in Social Media will be boarded. Simultaneously checking the popularity of these profiles, expressed in number of followers or fans is also checked. Through this study it is observed that, in general terms, the main journals of this field show a significant lack of interest towards the communication tool represented by social networking sites.
\end{abstract}

Keywords: Communication, Facebook, Journals, Social Networking Sites, Twitter

\section{Referencia normalizada}

SEGADO-BOJ, Francisco (2013): “¿Revistas 2.0? Revistas científicas españolas del área de Comunicación en las redes sociales”. Estudios sobre el mensaje periodístico. Vol. 19. Núm. especial abril, págs.: 1007-1016. Madrid, Servicio de Publicaciones de la Universidad Complutense.

Sumario: 1. Introducción: las redes sociales como ágoras de la nueva sociedad. 2. Objetivos y metodología. 3. Resultados; 3.1. Desinterés generalizado. Ranking de popularidad de las revistas científicas españolas de Comunicación en Facebook; 3.3. Ranking de popularidad de las revistas científicas españolas de Comunicación en Twitter. 4. Conclusiones; 4.1. ¿Usan las revistas de Comunicación las redes sociales?; 4.2. ¿Las revistas más seguidas son las que tienen un mayor índice de impacto?; 4.3. Discusión y futuras líneas de investigación. 5. Referencias bibliográficas.

\section{Introducción: las redes sociales como ágoras de la nueva sociedad}

Desde su aparición Internet se ha convertido en un "mecanismo de comunicación, de información y de dialogo interactivo entre los públicos y los emisores de los sitios web, para así convertir los internautas en públicos informados y en públicos activos" (Silva Robles y Elías Zambrano, 2011: 91). En este sentido la Web 2.0 "vuelve a dar protagonismo a la conversación social, impulsada por la metamorfosis profunda y

1 Trabajo íntegramente financiado por el Plan Propio de Investigación de la UNIR 
continua de las tecnologías de la comunicación" (Ruiz et al., 2010: 9). Dentro de la variedad de herramientas que han posibilitado el fenómeno que en su día Ben O'Reily etiquetó como web 2.0 (blogs, wikis, podcasts, etc...) "cabe destacar las redes sociales como aquellos que son capaces de aglutinar mayor número de usuarios y presentar las mejores cifras de audiencia y participación" (Abuín y Vinader, 2011: 9). Las redes sociales son "servicios web que permiten a los individuos (1) construer un perfil público o semi-público dentro de un sistema cerrado, (2) articular una lista de otros usuarios con los que comparte una conexión y (3) ver y navegar por su lista de contactos y de otras listas realizados por otros dentro del sistema" (Boyd y Ellison, 2007).

En la actualidad existen decenas o incluso centenas de redes sociales, generalistas como Google+, Myspace o Hi5, especializadas en un ámbito concreto como LinkedIn, centrada en las relaciones laborales o ResearchGate o Academia.Edu enfocadas al mundo científico y académico. De todas ellas la de mayor éxito es la norteamericana Facebook.

Nacida en 2004 como un proyecto interno para estudiantes de Harvard su éxito fue expandiéndola a otros centros universitarios de EEUU y acabó abriéndose al público general en 2006 (Phillips, 2007). En la actualidad sus 835 millones de usuarios registrados en todo el mundo ${ }^{2}$ sitúan a Facebook como la principal red social del mundo en la actualidad. De esa cifra, más de 16 millones son usuarios españoles lo que sitúa a España como el decimoquinto país donde esta red social logra una mayor penetración ${ }^{3}$.

El seguidor inmediato de Facebook en el ranking de popularidad de redes sociales es la plataforma de microblogging Twitter, que ocupa el segundo lugar de popularidad en el ranking de popularidad de redes sociales de EbizMBA ${ }^{4}$. Sin embargo, los ejecutivos de la compañía sus creadores se resisten a encasillar su servicio como una red social y se decantan por la etiqueta "red de noticias y conocimiento" (Acevedo, 2012), la bibliografía científica tradicionalmente incluye Twitter como otra red social más (Boyd y Ellison, 2007).

Este servicio fue fundado en 2006 por Jack Dorsey, Evan Williams y Biz Stone. Inspirados en la filosofía y el negocio de los SMS sus desarrolladores limitaron a 140 carácteres el texto de los mensajes que los usuarios podían publicar (Picard, 2011). En la actualidad cuenta con una cantidad global de usuarios que supera los quinientos millones (Dugan, 2012). En lo referente a España, más de cinco millones de personas poseen una cuenta en esta plataforma de microblogging (Nielsen y ComScore, en Moreno, 2012).

Estos datos implican que en estos momentos once de cada cien españoles son usuarios de Twitter mientras que treinta y cinco de cada cien tiene abierto un perfil en Facebook. Estos porcentajes dan idea de la importancia que las redes sociales cobran en

2 http://www.internetworldstats.com/facebook.htm. [Fecha de consulta: 29 de agosto de 2012].

$3 \mathrm{http}: / /$ www.socialbakers.com/facebook-statistics/. [Fecha de consulta: 29 de agosto de 2012].

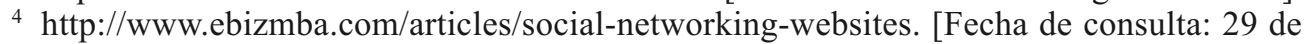
agosto de 2012]. 
España. Estas dimensiones convierten a las redes "en nuevos foros de actividad política y en nuevos canales de difusión de discursos y todo tipo de mensajes, tanto políticos como comerciales, o de servicios, institucionales, promocionales, empresariales, o publicitarios" (Túñez-López y Sixto-García, 2012: 30). Este nuevo espacio público que representan Internet y la redes sociales (Van Djick, 2012: 172) puede usarse pues para numerosos fines. El objetivo de esta investigación -como se desarrollará más adelante- consiste en comprobar si estas nuevas ágoras se usan también para la comunicación científica por parte de las revistas en España.

Al igual que Internet está produciendo cambios profundos e irreversibles en el periodismo (Edo, 2009, 9) las revistas académicas están sufriendo una transformación debida al impacto de Internet. Para muchas publicaciones científicas el número de visitas a su página web es un factor tan importante o más incluso que su tirada o distribución de ejemplares impresos (Weissmann, 2009). La presencia de las revistas científicas en las redes sociales "puede llegar a tener que ver con estrategias de notoriedad, relacionamiento y difusión" (Oller et al., 2012).

En España dos artículos han abordado recientemente la relación entre las revistas académicas y su presencia en las redes sociales. El primero de ellos ${ }^{5}$ realiza un análisis de del uso de Facebook que lleva a cabo una revista determinada: Revista Latina de Comunicación Social. Este estudio concluía que la presencia en las redes sociales de esta revista había contribuido a alcanzar un mayor índice de impacto.

Otro estudio más reciente ${ }^{6}$ analiza la presencia tanto en Twitter como en Facebook de todas las revistas recogidas en el Índice de impacto de las revistas españolas de Ciencias Sociales (IN-RECS) ${ }^{7}$. Este estudio concluye que "tan sólo un 4,7\% de las revista científicas de Ciencias Sociales se encuentran presentes en Facebook y un 2,5\% en Twitter" especificando que en el caso de Twitter "las revistas científicas del área de Comunicación muestran en proporción una mayor presencia encontrándose cerca del 25\%" (Oller, Segarra y Plaza, 2012: 65).

La presente investigación transcurre por el sendero iniciado por estos dos trabajos y profundiza en la presencia en el caso concreto de las revistas del campo de conocimiento de Comunicación.

\section{Objetivos y metodología}

Esta investigación plantea profundizar en la pregunta: "¿las revistas españolas de Comunicación están presentes en las redes sociales?" Como se ha mencionado en la introducción estas herramientas representan un vehículo de comunicación en auge y sería

5 HERRERO-GUTIÉRREZ, Francisco-Javier, et al. (2011): "Revista Latina de Comunicación Social en la red social Facebook", en Revista Latina de Comunicación Social, n 66: http://www.revistalatinacs.org/11/art/944_Salamanca/23_Javier.html. [fecha de consulta: 25 de junio de 2012].

6 OLLER ALONSO, Martín et al. (2012): "La presencia de las revistas científicas de Ciencias Sociales en los social media: de la web 1.0 a la web 2.0", en Index.comunicación, $\mathrm{n}^{\circ} 2$. Disponible en: http://journals.sfu.ca/indexcomunicacion/index.php/indexcomunicacion/article/download/27/32. [fecha de consulta: 25 de junio de 2012].

7 http://ec3.ugr.es/in-recs 
de esperar que las revistas académicas de esta área aprovechasen estas plataformas.

Para responder a esta cuestión se han localizado e identificado los perfiles que todas las revistas del IN-RECS categorizadas dentro del apartado de Comunicación poseen tanto en Facebook como en Twitter.

En primer lugar se ha consultado la web de las propias revistas para comprobar si enlazaban directamente a estas plataformas. En caso de no ser así se han utilizado los buscadores propios de estas dos plataformas. De no encontrar así resultados se ha utilizado Google para realizar la búsqueda "Nombre de la revista" + "Twitter"/“Facebook". En caso de aparecer múltiples resultados se ha acotado esta búsqueda mediante la fórmula: "Nombre de la revista" + "Nombre de la entidad editora" + "Twitter"/"Facebook".

Este procedimiento ha permitido trazar un mapa de la presencia de las principales revistas españolas de Comunicación tanto en Facebook como en Twitter.

Una vez identificadas estas revistas se ha procedido a un análisis cuantitativo de su popularidad tanto en Facebook como en Twitter. La segunda de las preguntas que ha querido responder este estudio es si existe una relación directa entre el índice de impacto obtenido por las revistas y su número de seguidores o fans. Dado que el índice de impacto se publica siempre con datos referidos al año anterior, no se puede calcular aún la influencia que la presencia en las redes sociales ejerce sobre el impacto o el número de cita. La pregunta se plantea para verificar la hipótesis de que las revistas con mayor impacto contarán con un mayor número de seguidores, dado que son las que despiertan mayor interés entre los investigadores.

Para responder a esta segunda respuesta se ha cuantificado un mismo día (el 15 de junio de 2012) el número de fans en Facebook y de seguidores en Twitter de estas revistas.

De manera concreta, se han planteado las siguientes hipótesis:

1) Las revistas científicas de Comunicación no utilizan las redes sociales.

2) Las revistas científicas con mayor índice de impacto son las más seguidas.

\section{Resultados}

\subsection{Desinterés generalizado}

De las 24 revistas indexadas en el In-Recs en su edición de 2010 cuatro de ellas gestionan un perfil en Facebook y siete poseen una cuenta oficial en Twitter.

Tabla 1: presencia de las revistas españolas de Comunicación en Facebook y Twitter.

Fuente: In-Recs y elaboración propia

\begin{tabular}{|l|c|c|c|c|}
\hline Revista & Cuartil & Índice impacto & Facebook & Twitter \\
\hline Latina & $1^{\circ}$ & 0,676 & Sí & Sí \\
\hline Comunicación y Sociedad & $1^{\circ}$ & 0,511 & No & Sí \\
\hline Comunicar & $1^{\circ}$ & 0,396 & Sí & Sí \\
\hline Estudios sobre el mensaje periodístico & $1^{\circ}$ & 0,213 & No & No \\
\hline Zer & $2^{\circ}$ & 0,174 & No & No \\
\hline Trípodos & $2^{\circ}$ & 0,169 & No & No \\
\hline Ámbitos & $2^{\circ}$ & 0,161 & No & No \\
\hline Análisi & $2^{\circ}$ & 0,087 & No & No \\
\hline
\end{tabular}




\begin{tabular}{|l|c|c|c|c|}
\hline Telos & $2^{\circ}$ & 0,087 & No & No \\
\hline Doxa & $3^{\circ}$ & 0,077 & No & Sí \\
\hline Sphera Publica & $3^{\circ}$ & 0,075 & No & No \\
\hline Área Abierta & $3^{\circ}$ & 0,071 & No & No \\
\hline Comunicación & $3^{\circ}$ & 0,050 & No & No \\
\hline Questiones publicitarias & $4^{\circ}$ & 0,045 & No & No \\
\hline Historia y Comunicación Social & $4^{\circ}$ & 0,043 & No & No \\
\hline Icono 14 & $4^{\circ}$ & 0,042 & No & Sí \\
\hline Cuadernos de Información y Comunicación CIC & $4^{\circ}$ & 0,027 & No & No \\
\hline Comunicación y hombre & $4^{\circ}$ & 0,000 & Sí & Sí \\
\hline Coneixement i societat & $4^{\circ}$ & 0,000 & No & No \\
\hline I/C Revista científica de información y comunicación & $4^{\circ}$ & 0,000 & No & No \\
\hline Mediaciones sociales & $4^{\circ}$ & 0,000 & Sí & Sí \\
\hline Pensar la publicidad & $4^{\circ}$ & 0,000 & No & No \\
\hline Revista de la SEECI & $4^{\circ}$ & 0,000 & No & No \\
\hline Vivat Academia & $4^{\circ}$ & 0,000 & No & Sí \\
\hline
\end{tabular}

El 70,83\% de las revistas científicas de este campo de conocimiento están ausentes de la web 2.0. Planteado de otro modo, sólo 3 de cada diez revistas científicas españolas se han planteado utilizar las redes sociales para aumentar la visibilidad de los resultados de investigación que publican. Las revistas que sí emplean estos medios de comunicación se decantan mayoritariamente por Twitter (siete revistas) y sólo cuatro cuentan con un perfil en Facebook. Resulta interesante comprobar cómo las cuatro revistas presentes en Facebook están también presentes en Twitter.

\subsection{Ranking de popularidad de las revistas científicas españolas de Comunica- ción en Facebook}

La revista líder en Facebook es Revista Latina de Comunicación Social, que en el momento de realizar este estudio contaba con 2.224 fans, una cantidad que casi triplicaba los 780 fans de su más inmediata competidora, Comunicar (ver Gráfico 1).

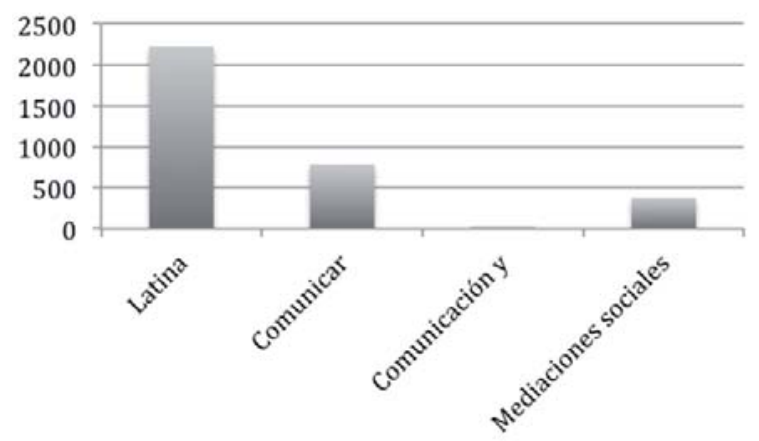

Gráfico 1. Número de fans de las revistas españolas de Comunicación en Facebook (15-6-2012). Elaboración propia. 
El tercer puesto es ocupado por Mediaciones sociales con 373 fans. Comunicación y hombre se sitúa en último lugar con un resultado casi marginal de 23 fans.

\subsection{Ranking de popularidad de las revistas científicas españolas de Comunica- ción en Twitter}

El mayor número de seguidores en Twitter (1.422) es logrado por la cuenta de Comunicación y sociedad. El segundo y tercer puesto corresponden respectivamente a Comunicar (738) y a Revista Latina de Comunicación Social (501).

De las cinco revistas restantes tan sólo dos consiguen superar el centenar de followers. Se trata de Icono 14 (180 seguidores) y de Mediaciones sociales (140).

Las otras tres revistas se limitan a ser seguidas por 54 usuarios (Comunicación y hombre), 14 (Doxa) y 8 (Vivat Academia) usuarios (ver Gráfico 2).

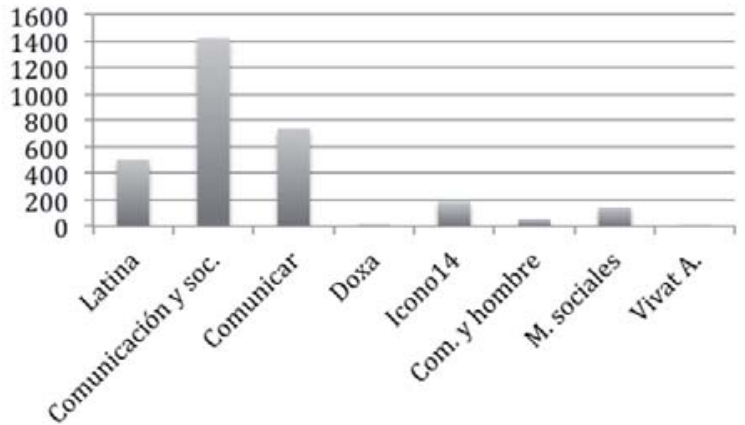

Gráfico 2. Número de seguidores de las revistas españolas de Comunicación en Twitter (15-6-2012). Elaboración propia.

\section{Conclusiones}

Los datos obtenidos por el estudio y que acaban de presentarse permiten responder a las hipótesis planteadas.

\section{1. ¿Usan las revistas de Comunicación las redes sociales?}

A la vista de los datos obtenidos (recordemos, sólo un 30\% de las revistas cuentan con un perfil en Facebook o en Twitter) la respuesta es clara: la gran mayoría, no. Además se puede señalar que el desinterés por las redes sociales es mayor precisamente hacia la más importante de ellas. Tan sólo un 17\% de estas revistas mantiene un perfil en Facebook.

Puede asegurarse entonces que las revistas españolas cometen el mismo error que una experta en social media atribuía a muchos empresarios: "Guiados por hechos como la enorme cantidad de estados y tweets que se dedican a comentar detalles insignificantes de la vida cotidiana, tendencias en Twitter sobre escándalos mediáticos, o actualizaciones en Facebook sobre los juegos en los que sus contactos participan on-line, algunos empresarios pueden creer que no se trata de una forma seria de hacer negocios. Pero con más de 840 millones de usuarios en Facebook y de 140 millones en Twitter [...] se puede decir que hay lugar para todo, tanto para el esparcimiento como para los negocios" (Moschini, 2012). Donde la autora citada habla de "negocios", nosotros po- 
demos hablar de "conocimiento". De hecho, en las investigaciones sobre el uso de redes sociales en el ámbito docente coinciden en señalar el enorme potencial de estas herramientas -incluida Facebook- aparte de su uso como espacio de comunicación, información y entretenimiento (Gómez Aguilar et al., 2012: 132). Incluso desde el área de la bibliometría se señala que difundiendo el trabajo científico "con las herramientas de la Web social cobra una nueva vida con una mayor audiencia potencial y un público más diversificado (Torres-Salinas y Delgado-López-Cózar, 2009: 539). Las revistas españolas en Comunicación aún tienen que aprender esta lección.

\section{2. ¿Las revistas más seguidas son las que tienen un mayor índice de impacto?}

La respuesta en esta ocasión es un rotundo sí. Las revistas del primer cuartil presentes en Facebook (Latina y Comunicar) y en Twitter (Comunicación y Sociedad, Comunicar y Latina) se sitúan a una distancia abismal del resto de revistas.

En el caso de Facebook la revista menos seguida de primer cuartil (Comunicar) casi duplica en fans a la siguiente revista en número de fans (Mediaciones sociales): 780 frente a 373. En Twitter se repite una situación casi idéntica. La revista del primer cuartil con un menor número de followers (Latina) casi triplica a la siguiente en el escalafón en cuanto a seguidores (Icono 14): 501 frente a 184.

\subsection{Discusión y futuras líneas de investigación}

Como se acaba de señalar, entre las revistas científicas de Comunicación se da un desinterés generalizado respecto a su presencia en las redes sociales. Respecto a los usuarios se ha comprobado que de manera general optan por seguir a las revistas con mayor impacto del área.

Para el futuro, cuando se publiquen los datos de impacto de 2012, resultaría de interés comprobar si la presencia de las revistas ajenas al primer cuartil en Facebook y Twitter desemboca en un mayor índice de citación como consecuencia de la visibilidad que les ofrecen estas redes, aunque solo sea por comparación respecto a las revistas ausentes. Del mismo modo, podría comprobarse la presencia y uso de publicaciones científicas que de momento no han conseguido hacerse un hueco en el IN-RECS. En este sentido podría estudiarse el uso que las cabeceras que integran la Plataforma Latina de Revistas de Comunicación ${ }^{8}$ (Miguel Hernández Communication Journal, Revista Mediterránea de Comunicación, Fonseca Journal of Communication, Pangea, Fotocinema, TecCom Studies, Communication Papers, Index.Comunicación y Revista de Cibercomunicación), un proyecto impulsado en 2010 desde la Sociedad Latina de Comunicación Social con el objetivo de que las revistas existentes apadrinaran la aparición de nuevas publicaciones que ampliaran la escasa población de revistas académicas españolas sobre comunicación (Herrero et al., 2012: 70-71). Este estudio podría incluir también cualesquiera otras revistas ajenas al IN-RECS (Derecom o Revista Internacional de Relaciones Públicas, por citar sólo dos ejemplos). Este análisis permitiría determinar si la juventud de las revistas o su au-

$8 \mathrm{http}: / /$ www.plataformarevistascomunicacion.org/quiene-somos/. Consultado el 30 de agosto de 2012. 
sencia del IN-RECS constituyen factores determinantes para su presencia en redes sociales.

Resultaría necesario asimismo determinar hasta qué punto el índice de impacto del IN-RECS constituye el único factor influyente en la popularidad de estas revistas dentro de las redes sociales o si por el contrario el ritmo de actualización o el tipo de mensajes que se publican afectan al número de seguidores. Así pues, por ejemplo, podría investigarse si las revistas con mayor actividad dentro de un mismo cuartil del INRECS atraen a un mayor número de fans o followers.

En un sentido similar podría analizarse la presencia en Facebook y Twitter de las revistas de Comunicación presentes en otras bases de datos internacionales como Scopus o el Social Sciencies Citation Index. Esto permitiría verificar si el desinterés generalizado desvelado por este estudio se da también fuera de las fronteras españolas.

Por último podría y debería abordarse en futuros análisis la presencia de las revistas científicas del área en redes sociales centradas específica y únicamente en el campo académico como ResearchGate, Mendeley o Academia.edu. Así se podría comprobar si la existencia de plataformas específicamente académicas resulta más atractiva a las revistas que otras a las que se considera únicamente aptas para contenidos informales.

\section{Referencias bibliográficas}

ABUÍN, Natalia y VINADER, Raquel (2011): "El desarrollo de la World Wide Web en España: una aproximación teórica desde sus orígenes hasta su transformación en un medio semántico", en Razón y Palabra, no 75: http://www.razonypalabra .org.mx/N/N75/varia_75/varia3parte/31_Avuin_V75.pdf. [fecha de consulta: 25 de junio de 2012]

ACEVEDO, Inti (2012): “10 grandes mitos de Twitter", en Alt1040: http://alt1040 .com/2012/06/10-grandes-mitos-de-twitter. [fecha de consulta: 2 de julio de 2012]

BOYD, Danah M. y ELLISON, Nichol B. (2007): "Social Network Sites: Definition, history and scholarship", en Journal of Computer-Mediated Communication, vol. 13, $\mathrm{n}^{\mathrm{o}} 1$ : http://jcmc.indiana.edu/voll3/issue1/boyd.ellison.html. [fecha de consulta: 2 de julio de 2012]

DUGAN, Lauren (2012): "Twitter to surpass 500 million registered users on Wednesday", en All Twitter: http://www.mediabistro.com/alltwitter/500-million-registeredusers_b18842 [fecha de consulta: 28 de agosto de 2012]

EDO BOLÓS, Concepción (2009): Periodismo informativo e interpretativo. El impacto de Internet en la noticia, las fuentes y los géneros. Sevilla, Comunicación Social.

GÓMEZ AGUILAR, Gema et al. (2012): "El uso académico de las redes sociales en universitarios", en Comunicar, no 38, pp. 131-138: http://www.revistacomunicar.com/index.php? contenido $=$ detalles \&numero $=38 \&$ articulo $=38-2012-16[$ fecha de consulta: 4 de agosto de 2012]

HERRERO-GUTIÉRREZ, Francisco-Javier et al. (2011): "Revista Latina de Comunicación Social en la red social Facebook", en Revista Latina de Comunicación 
Social, $\mathrm{n}^{\circ}$ 66. Disponible en: http://www.revistalatinacs.org/11/art/944_Salamanca/23_Javier.html. [fecha de consulta: 25 de junio de 2012]

HERRERO GUTIÉRREZ, Javier, et al. (2012): “Análisis cibermétrico de cinco revistas emergentes de comunicación en sus dos primeros años en línea: Revista Mediterránea de Comunicación, Fonseca Journal of Communication, Miguel Hernández Communication Journal, Revista Pangea y Fotocinema", en Index.Comunicación, $\mathrm{n}^{\circ} 2$ : http://journals.sfu.ca/indexcomunicacion/index.php/indexcomunicacion/article/view/32/36. [fecha de consulta: 30 de agosto de 2012]

MORENO, Manuel (2012): "Twitter supera los 15 millones de usuarios en España", en Trecebits.com: http://www.trecebits.com/2012/07/02/twitter-supera-los-5-millones-de-usuarios-en-espana/ [fecha de consulta: 15 de julio de 2012]

MOSCHINI, Silvina (2012): "Mitos sobre el marketing en redes sociales", en La Vanguardia, 27 de agosto: http://www.lavanguardia.com/economia/marketing-publicidad/20120827/54342348640/mitos-sobre-el-marketing-en-redes-sociales.html [fecha de consulta: 28 de agosto de 2012]

OLLER ALONSO, Martín et al.: "La presencia de las revistas científicas de Ciencias Sociales en los social media: de la web 1.0 a la web 2.0", en Index.comunicación, $\mathrm{n}^{\mathrm{o}} 2$ : http://journals.sfu.ca/indexcomunicacion/index.php/indexcomunicacion/article/download/27/32 [fecha de consulta: 25 de junio de 2012]

PHILLIPS, Sarah (2007): “A brief history of Facebook", en The Guardian, 25 de Julio: http://www.guardian.co.uk/technology/2007/jul/25/media.newmedia. [fecha de consulta: 28 de julio de 2012]

PICARD, André (2011): "The history of Twitter: 140 characters at a time", en The Globe and Mail, 20 de marzo: http://www.theglobeandmail.com/technology/digital-culture/social-web/the-history-of-twitter-140-characters-at-a-time/article573416/. [fecha de consulta: 28 de agosto de 2012]

RUIZ, Carlos et al. (2010): "Conversación 2.0 y democracia. Análisis de los comentarios de los lectores en la prensa digital catalana", en Comunicación y Sociedad, vol. XXII, $\mathrm{n}^{\circ}$ 2: http://www.unav.es/fcom/comunicacionysociedad/es/resumen.php ?art id $=360$ [fecha de consulta: 14 de agosto de 2012]

SILVA ROBLES, Carmen y ELÍAS ZAMBRANO, Rodrigo (2011): "Relaciones públicas 2.0 (y Educomunicación) ¿De qué hablamos realmente? Un acercamiento conceptual y estratégico", en Fonseca, Journal of Communication, $\mathrm{n}^{\mathrm{o}} 3$ : http://fjc.usal.es/index.php/sumario3/77-relacionespublicas20 fecha de consulta: 18 de julio de 2012]

TORRES-SALINAS, Daniel y DELGADO-LÓPEZ-CÓZAR, Emilio (2009): “Estrategia para mejorar la difusión de los resultados de investigación con la Web 2.0", en El profesional de la Información, v.18, n5, pp. 534-539.

TÚÑEZ-LÓPEZ, Miguel y SIXTO-GARCÍA, José (2012): “Un escaño en Facebook: Política 2.0, marketing viral y redes sociales", en Vivat Academia, $\mathrm{n}^{\mathrm{o}} 118$ : http://www.seeci.net/vivataca/numeros/n118/PDFs/Varios1.pdf [fecha de consulta: 1 de septiembre de 2012] 
VAN DJICK, José (2012): "Facebook as a tool for producing sociality and connectivity", en Television and new media, vol. 13, n' 2, pp. 160-176.

WEISSMANN, Gerald (2009): "Walter Benjamin and Biz Stone: The scientific paper in the age of Twitter", en The FASEB Journal, vol. 23, nº 7: http://www.fasebj.org /content/23/7/2015.full [fecha de consulta: 12 de julio de 2012]

\section{Francisco SEGADO-BOJ}

Universidad Internacional de la Rioja

Grupo de Investigación "Comunicación y Sociedad Digital"

Profesor Adjunto

francisco.segado@unir.net 